\title{
INFINITE TRANSITIVITY ON AFFINE VARIETIES
}

\author{
I. ARZHANTSEV, H. FLENNER, S. KALIMAN, F. KUTZSCHEBAUCH, M. ZAIDENBERG
}

\begin{abstract}
In this note we survey recent results on automorphisms of affine algebraic varieties, infinitely transitive group actions and flexibility. We present related constructions and examples, and discuss geometric applications and open problems.
\end{abstract}

\section{INTRODUCTION}

An action of a group $G$ on a set $A$ is said to be $m$-transitive if for every two tuples of pairwise distinct points $\left(a_{1}, \ldots, a_{m}\right)$ and $\left(a_{1}^{\prime}, \ldots, a_{m}^{\prime}\right)$ in $A$ there exists $g \in G$ such that $g \cdot a_{i}=a_{i}^{\prime}$ for all $i=1, \ldots, m$. An action which is $m$-transitive for all $m \in \mathbb{Z}_{>0}$ will be called infinitely transitive.

Clearly, the group of all bijections of an infinite set $A$ acts infinitely transitively on $A$. Infinite transitivity never occurs if $G$ is a Lie group or algebraic group acting on a variety $A$. Indeed, $m$-transitivity implies that the map $G \rightarrow A^{m}$ with $g \mapsto\left(g . a_{1}, \ldots, g . a_{m}\right)$ is dominant for an $m$-tuple of pairwise distinct points $\left(a_{1}, \ldots, a_{m}\right) \in A^{m}$. This shows that $G$ cannot act on $A m$-transitively if $\operatorname{dim} G<m \cdot \operatorname{dim} A$. According to A. Borel a much stronger result is valid: a real Lie group can not act even 3-transitively on a simply connected, non-compact real manifold (see Theorems 5 and 6 in [5]). By a result of F. Knop [18], the most transitive action of an algebraic group over an algebraically closed field is the 3 -transitive action of the group $\mathrm{PSL}_{2}$ on the projective line $\mathbb{P}^{1}$.

At the same time, the group $\operatorname{Aut}\left(\mathbb{A}^{n}\right)$ of all algebraic automorphisms of the affine space $\mathbb{A}^{n}$ over an infinite field acts infinitely transitively on $\mathbb{A}^{n}$ for $n \geq 2$. To obtain this result, it suffices to use linear automorphisms and triangular automorphisms of the form

$$
\left(x_{1}, \ldots, x_{n-1}, x_{n}\right) \mapsto\left(x_{1}, \ldots, x_{n-1}, x_{n}+P\left(x_{1}, \ldots, x_{n-1}\right)\right),
$$

where $P\left(x_{1}, \ldots, x_{n-1}\right)$ is an arbitrary polynomial. These automorphisms generate the tame automorphism group TAut $\left(\mathbb{A}^{n}\right)$, which acts infinitely transitively on $\mathbb{A}^{n}$ for $n \geq 2$.

It was shown in [1] that for certain (infinite dimensional) groups of automorphisms of affine varieties transitivity implies infinite transitivity. We do not try to present here the results of [1] in full generality, but rather to concentrate on the most interesting features.

\section{MAin Results}

Let $X$ be an algebraic variety over a field $\mathbb{k}$. Unless we explicitly precise the opposite, we assume usually that $\mathbb{k}$ is algebraically closed of characteristic zero. Consider a regular action $\mathbb{G}_{a} \times X \rightarrow X$ of the additive group $\mathbb{G}_{a}=(\mathbb{k},+)$ of the ground field on $X$. The image, say, $H$ of $\mathbb{G}_{a}$ in the automorphism group $\operatorname{Aut}(X)$ is a one-parameter

2010 Mathematics Subject Classification: 14R20,32M17; secondary 14L30. Key words: affine variety, group action, one-parameter subgroup, transitivity. 
unipotent subgroup of $\operatorname{Aut}(X)$. We let $\operatorname{SAut}(X)$ denote the subgroup of $\operatorname{Aut}(X)$ generated by all its one-parameter unipotent subgroups. Automorphisms from the group $\operatorname{SAut}(X)$ will be called special. In general, $\operatorname{SAut}(X)$ is a normal subgroup of $\operatorname{Aut}(X)$.

Denote by $X_{\text {reg }}$ the smooth locus of an algebraic variety $X$. We say that a point $x \in X_{\text {reg }}$ is flexible if the tangent space $T_{x} X$ is spanned by the tangent vectors to the orbits $H \cdot x$ over all one-parameter unipotent subgroups $H$ in $\operatorname{Aut}(X)$. The variety $X$ is flexible if every point $x \in X_{\text {reg }}$ is. Clearly, $X$ is flexible if one point of $X_{\text {reg }}$ is and the group $\operatorname{Aut}(X)$ acts transitively on $X_{\text {reg. }}$.

The following result conjectured in [2] is proven in [1, Theorem 0.1].

Theorem 1.1. Let $X$ be an irreducible affine variety of dimension $\geq 2$. Then the following conditions are equivalent.

(1) The group $\operatorname{SAut}(X)$ act transitively on $X_{\text {reg }}$.

(2) The group $\operatorname{SAut}(X)$ act infinitely transitively on $X_{\text {reg }}$.

(3) The variety $X$ is flexible.

\section{EXAMPLES OF FLEXIBLE VARIETIES}

We are going to show that the equivalent conditions of Theorem 1.1 are satisfied for wide classes of affine varieties.

2.1. Suspensions. Let $X$ be an affine variety. Given a nonconstant regular function $f \in \mathbb{k}[X]$, we define a new affine variety

$$
\operatorname{Susp}(X, f)=\{u v-f(x)=0\} \subseteq \mathbb{A}^{2} \times X
$$

called a suspension over $X$. It is shown in [2, Theorem 3.2] that a suspension over a flexible affine variety is again flexible. The case of suspensions over affine spaces was treated earlier in [14]. Iterating the construction of suspension yields new examples of flexible varieties.

Flexibility and infinite transitivity of the action of $\operatorname{SAut}(X)$ is established in $[2$, Theorem 3.1] for a suspension $X=\{u v-f(x)=0\}$ over the affine line $\mathbb{A}^{1}$ under the assumption that $f(\mathbb{k})=\mathbb{k}$, where $\mathbb{k}$ is an arbitrary field of characteristic zero. The same holds for suspensions over flexible real affine algebraic varieties with connected smooth loci ([2, Theorem 3.3]). By [19], infinite transitivity holds on every connected component of the smooth loci of suspensions over flexible real affine varieties.

2.2. Affine toric varieties. Recall that a normal algebraic variety $X$ is toric if it admits a regular action of an algebraic torus $T$ with an open orbit. In general, an affine toric variety does not need to be flexible. For instance, if $X=T$ then the algebra $\mathbb{k}[X]$ is generated by invertible functions and hence the group $\operatorname{SAut}(X)$ is trivial.

We say that an affine toric variety $X$ is nondegenerate if the only invertible regular functions on $X$ are nonzero constants. Equivalently, $X$ is nondegenerate if it is not isomorphic to $X^{\prime} \times\left(\mathbb{A}^{1} \backslash\{0\}\right)$ for some toric variety $X^{\prime}$. By [2, Theorem 2.1] any nondegenerate affine toric variety is flexible. Considering affine toric surfaces, one obtains examples of affine varieties $X$ such that $X_{\text {reg }}$ is not a homogeneous space of an algebraic group, but the group $\operatorname{SAut}(X)$ acts on $X_{\text {reg }}$ infinitely transitively, see [2, Example 2.2]. 
2.3. Homogeneous spaces. Let us consider (following [24]) the class of connected linear algebraic groups $G$ generated by one-parameter unipotent subgroups. A connected linear algebraic group $G$ belongs to this class if and only if $G$ does not admit non-trivial characters, or, equivalently, if a maximal reductive subgroup of $G$ is semisimple. If such a group $G$ acts on a variety $X$ then the image of $G$ in $\operatorname{Aut}(X)$ is contained in $\operatorname{SAut}(X)$. If $G$ acts on $X_{\text {reg }}$ transitively then $X$ is flexible.

As an example, consider a simple rational $G$-module $V$, where $G$ is semisimple. The cone $X$ of highest weight vectors in $V$ consists of two $G$-orbits, namely, the open orbit $X \backslash\{0\}$ and the origin $\{0\}$ ([25]). If $X \neq V$ then $G$ acts on $X_{\text {reg }}$ transitively, hence the group $\operatorname{SAut}(X)$ is infinitely transitive on $X_{\text {reg. }}$. Note that $X$ may be considered as a (normal) affine cone over the flag variety $G / P$, where a parabolic subgroup $P$ is the stabilizer of a point in the projectivization $\mathbb{P}(X)$ of the cone $X$ in $\mathbb{P}(V)$. In these terms infinite transitivity for $X$ was proven in [2, Theorem 1.1].

Any affine homogeneous space $G / H$ of dimension $\geq 2$ satisfies the equivalent conditions of Theorem 1.1 provided that $G$ does not admit non-trivial characters, see [1, Proposition 5.4]. In particular, for any semisimple group $G$ and a reductive subgroup $H \subseteq G$ the homogeneous space $X=G / H$ is flexible and the group $\operatorname{SAut}(X)$ is infinite transitive on $X$. This applies as well to $X=G$.

2.4. Almost homogeneous varieties. Suppose that a connected semisimple algebraic group $G$ acts with an open orbit on an irreducible affine variety $X$. In this case we say that $X$ is almost homogeneous. It turns out that under some additional assumptions this implies flexibility of $X$.

2.4.1. The smooth case. Assume that an almost homogeneous affine variety $X$ is smooth. Using Luna's Étale Slice Theorem we show in [1, Theorem 5.6] that $X$ is homogeneous under the action of a semidirect product $G \ltimes V$, where $V$ is a certain finite-dimensional $G$-module. In particular $X$ is flexible.

2.4.2. $\mathrm{SL}_{2}$-embeddings. Let the group $\mathrm{SL}_{2}=\mathrm{SL}_{2}(\mathbb{k})$ acts with an open orbit on a normal affine threefold $X$. All such $\mathrm{SL}_{2}$-threefolds were classified in [23]. If $X$ is smooth then it is flexible by the above argument. For a singular $X$ the complement of the open $\mathrm{SL}_{2}$-orbit consists of a two-dimensional orbit, say, $O$ and a singular fixed point $p \in X$.

It is shown in [3] that $X$ can be obtained as the quotient of an affine hypersurface $x_{0}^{b}=x_{1} x_{4}-x_{2} x_{3}$ under an action of a one-parameter diagonalizable group. Such a hypersurface is a suspension over $\mathbb{A}^{3}$. Using this one can join a point in $O$ with a point in the open $\mathrm{SL}_{2}$-orbit by a $\mathbb{G}_{a}$-orbit on $X$ and thus to gain flexibility of $X$; see [1, Theorem 5.7] for details.

2.5. Vector bundles. Let $\pi: E \rightarrow X$ be a reduced, irreducible linear space over a flexible variety $X$, which is a vector bundle over $X_{\text {reg }}$. Assume that there is an action of the group $\operatorname{SAut}(X)$ on $E$ such that the action of every one-parameter unipotent subgroup is algebraic and the morphism $\pi$ is equivariant. It is shown in [1, Corollary 4.5] that the total space $E$ is a flexible variety. In particular, the tangent bundle $T X$ and all tensor bundles $E=(T X)^{\otimes a} \otimes\left(T^{*} X\right)^{\otimes b}$ are flexible.

2.6. Affine cones over projective varieties. Let $X$ be the affine cone over a projective variety $Y$ polarized by a very ample divisor $H$. Then one can characterize 
flexibility of $X$ in terms of certain geometric properties of the pair $(Y, H)$ as follows (see [22]).

An open subset $U \subseteq Y$ is called a cylinder if $U \cong Z \times \mathbb{A}^{1}$, where $Z$ is a smooth affine variety. A cylinder $U$ is called $H$-polar if $U=Y \backslash \operatorname{Supp} D$ for some effective $\mathbb{Q}$-divisor $D$ linearly equivalent to $H([16])$. It is shown in [16, Theorem 3.9] that any $H$-polar cylinder $U$ on $Y$ gives rise to a $\mathbb{G}_{a}$-action on the affine cone $X$ over $Y$.

A subset $W \subseteq Y$ is called invariant with respect to a cylinder $U \cong Z \times \mathbb{A}^{1}$ if $W \cap U=\pi^{-1}(\pi(W))$, where $\pi: U \rightarrow Z$ is the first projection. In other words, $W$ is invariant if every $\mathbb{A}^{1}$-fiber of the cylinder is either contained in $W$ or does not meet $W$. A variety $Y$ is transversally covered by cylinders $U_{i}, i=1, \ldots, s$, if $Y=\bigcup_{i} U_{i}$ and there is no proper subset $W \subseteq Y$ invariant with respect to all the $U_{i}$.

Theorem 2.5 in [22] states that if for some very ample divisor $H$ on a normal projective variety $Y$ there exists a transversal covering by $H$-polar cylinders then the corresponding affine cone $X$ over $Y$ is flexible. This criterion allows to establish that any affine cone over a del Pezzo surface of degree $\geq 5$ is flexible. The same is true for certain affine cones over del Pezzo surfaces of degree 4, including the pluri-anticanonical ones. In contrast, the pluri-anticanonical cones over del Pezzo surfaces of degree 1 or 2 do not admit any non-trivial action of a unipotent algebraic group, neither any effective action of a two-dimensional connected algebraic group ([17]). The case of cubic surfaces remains open.

2.7. Gizatullin surfaces. These are normal affine surfaces which admit a completion by a chain of smooth rational curves. It follows from Gizatullin's Theorem ([12, Theorems 2 and 3], see also [7]) that a normal affine surface $X$ different from $\mathbb{A}^{1} \times\left(\mathbb{A}^{1} \backslash\{0\}\right)$ is Gizatullin if and only if the special automorphism group $\operatorname{SAut}(X)$ has an open orbit; then this open orbit necessarily has a finite complement in $X$. It was conjectured in [12] that if the base field $\mathbb{k}$ has characteristic zero then the open $\operatorname{SAut}(X)$-orbit coincides with $X_{\text {reg }}$ i.e. that

every Gizatullin surface is flexible.

This is definitely not true in a positive characteristic, where the automorphism group Aut $(X)$ of a Gizatullin surface $X$ can have fixed points that are smooth points of $X$ [6]. We have seen in 2.1 that Gizatullin's Conjecture is true for the Gizatullin surfaces given in $\mathbb{A}^{3}$ by equations $x y-f(z)=0$, since these are suspensions of the affine line. Yet another class of flexible Gizatullin surfaces consists of the DanilovGizatullin surfaces, see [11]. Recently S. Kovalenko constructed a counterexample to the Gizatullin Conjecture over $\mathbb{C}$ (unpublished).

We refer the reader to [9] and the references therein for a study of one-parameter groups acting on Gizatullin surfaces.

\section{TECHNiCAL TOOLS}

We do not try to expose the proof of Theorem 1.1 in detail. In this section we just present a couple of technical tools which play a crucial role in the proof. The first one is the well known correspondence between regular $\mathbb{G}_{a}$-actions on an affine variety $X$ and locally nilpotent derivations of the algebra $A=\mathbb{k}[X]$ of regular functions on $X$. 
3.1. Locally nilpotent derivations and their replicas. A derivation $\partial$ of an algebra $A$ is called locally nilpotent if for any $a \in A$ there exists $m \in \mathbb{Z}_{>0}$ such that $\partial^{m}(a)=0$. If the group $\mathbb{G}_{a}$ acts on $X=\operatorname{Spec} A$ then the associated derivation $\partial$ of $\mathrm{A}$ is locally nilpotent. It is immediate that for every $f \in \operatorname{ker} \partial$ the derivation $f \partial$ is again locally nilpotent.

Conversely, given a locally nilpotent derivation $\partial: A \rightarrow A$ and $t \in \mathbb{k}$, the map $\exp (t \partial): A \rightarrow A$ is an automorphism of A. Furthermore for $\partial \neq 0, H=\exp (t \partial)$ is a one-parameter unipotent subgroup of $\operatorname{Aut}(A)$. Via the isomorphism $\operatorname{Aut}(A) \cong \operatorname{Aut}(X)$ given by $g \rightarrow\left(g^{-1}\right)^{*}$ this yields a one-parameter unipotent subgroup of $\operatorname{Aut}(X)$, which we denote by the same letter $H$. We refer to [10] for more details on locally nilpotent derivations.

The algebra of invariants $\mathbb{k}[X]^{H}=\operatorname{ker} \partial$ has transcendence degree $\operatorname{dim} X-1$ over $\mathbb{k}$. Given an invariant $f \in \mathbb{k}[X]^{H}$ the one-parameter unipotent subgroup $H_{f}=\exp (\mathbb{k} f \partial)$, called a replica of $H$, plays an important role in the sequel. The $H_{f}$-action has the same general orbits as the $H$-action. However, the zero locus of $f$ remains pointwise fixed under the $H_{f}$-action. So given a finite set of points chosen on distinct general $H$-orbits one can find a replica $H_{f}$ of $H$ that moves all the points but a given one. If we have at our disposal enough $\mathbb{G}_{a}$-actions in transversal directions on $X$ then by changing the velocity along the corresponding orbits as above, we can move the given ordered finite set in $X_{\text {reg }}$ into a prescribed position. This gives the infinite transitivity of the $\operatorname{SAut}(X)$-action on $X_{\text {reg. }}$.

Let us illustrate the notions of a replica and of a special automorphism in the case of an affine space $\mathbb{A}^{n}$ over $\mathbb{k}$. The group $\operatorname{SAut}\left(\mathbb{A}^{n}\right)$ contains the one-parameter unipotent subgroup of translations in any given direction. The infinitesimal generator of such a subgroup is a directional partial derivative. Such a derivative defines a locally nilpotent derivation of the polynomial ring in $n$ variables, whose phase flow is the group of translations in this direction. Its replicas are the one parameter groups of shears in the same direction.

As another example, consider the locally nilpotent derivation $\partial=X \frac{\partial}{\partial Y}+Y \frac{\partial}{\partial Z}$ of the polynomial ring $\mathbb{k}[X, Y, Z]$ and an invariant function $f=Y^{2}-2 X Z \in \operatorname{ker} \partial$. The corresponding replica $H_{f}$ contains in particular the famous Nagata automorphism $H_{f}(1)=\exp (f \cdot \partial) \in \operatorname{SAut}\left(\mathbb{A}^{3}\right)$, which is known to be wild; see [29].

Notice that any automorphism $\alpha \in \operatorname{SAut}\left(\mathbb{A}^{n}\right)$ preserves the usual volume form on $\mathbb{A}^{n}$. Hence $\operatorname{SAut}\left(\mathbb{A}^{n}\right) \subseteq G_{n}$, where $G_{n}$ denotes the subgroup of $\operatorname{Aut}\left(\mathbb{A}^{n}\right)$ consisting of all automorphisms with Jacobian determinant 1 . The problem whether the subgroup $\operatorname{SAut}\left(\mathbb{A}^{n}\right)$ coincides with $G_{n}$ is widely open. Recall that this is the case in dimension 2 due to the Jung-van der Kulk Theorem.

Algebraically generated groups. Our second tool is a technique to work with infinite dimensional groups. We say that a subgroup $H$ of the automorphism group $\operatorname{Aut}(X)$ is algebraic if $H$ has a structure of an algebraic group such that the natural action $H \times X \rightarrow X$ is a morphism. A subgroup $G$ of $\operatorname{Aut}(X)$ is called algebraically generated if it is generated as an abstract group by a family $\mathcal{G}$ of connected algebraic subgroups of $\operatorname{Aut}(X)$. Similar notions were studied in the literature earlier, see e.g. [26], [28], and more recently [24]. 
In [1] we extend some standard facts on finite-dimensional algebraic transformation groups to the case of algebraically generated groups. It is not difficult to show that for any point $x \in X$ the orbit $G \cdot x$ is locally closed. What is more surprising, one can find (not necessarily distinct) subgroups $H_{1}, \ldots, H_{s} \in \mathcal{G}$ such that

$$
G . x=\left(H_{1} \cdot H_{2} \cdot \ldots \cdot H_{s}\right) \cdot x
$$

for any $x \in X$, see [1, Proposition 1.5].

In our setting we obtain the following version of Kleiman's Transversality Theorem [1, Theorem 1.15].

Theorem 3.1. Let a subgroup $G \subseteq \operatorname{Aut}(X)$ be algebraically generated by a system $\mathcal{G}$ of connected algebraic subgroups closed under conjugation in $G$. Suppose that $G$ acts with an open orbit $O \subseteq X$. Then there exist subgroups $H_{1}, \ldots, H_{s} \in \mathcal{G}$ such that for any locally closed reduced subschemes $Y$ and $Z$ in $O$ one can find a Zariski dense open subset $U=U(Y, Z) \subseteq H_{1} \times \ldots \times H_{s}$ such that every element $\left(h_{1}, \ldots, h_{s}\right) \in U$ satisfies the following.

(a) The translate $\left(h_{1} \cdot \ldots \cdot h_{s}\right) \cdot Z_{\text {reg }}$ meets $Y_{\text {reg }}$ transversally.

(b) $\operatorname{dim}\left(Y \cap\left(h_{1} \cdot \ldots \cdot h_{s}\right) \cdot Z\right) \leq \operatorname{dim} Y+\operatorname{dim} Z-\operatorname{dim} X$.

In particular $Y \cap\left(h_{1} \cdot \ldots \cdot h_{s}\right) \cdot Z=\varnothing$ if $\operatorname{dim} Y+\operatorname{dim} Z<\operatorname{dim} X$.

The next generalization concerns the Rosenlicht Theorem on invariants. It turns out that for any algebraically generated subgroup $G \subseteq \operatorname{Aut}(X)$ there exists a finite collection of rational $G$-invariants on $X$ which separate $G$-orbits in general position [1, Theorem 1.13]. In particular, the codimension of a general $G$-orbit in $X$ equals the transcendence degree of the field $\mathbb{k}(X)^{G}$ of rational $G$-invariants over $\mathbb{k}$. The latter result has a useful corollary.

The Makar-Limanov invariant. Recall [10] that the Makar-Limanov invariant $\operatorname{ML}(X)$ of an affine algebraic variety $X$ is the intersection of the kernels of all locally nilpotent derivations on $\mathbb{k}[X]$. In other words, $\operatorname{ML}(X)$ is the subalgebra of all $\operatorname{SAut}(X)$-invariants of the algebra $\mathbb{k}[X]$. Similarly [21] the field Makar-Limanov invariant $\operatorname{FML}(X)$ is defined as the intersection of the kernels of extensions of all locally nilpotent derivations on $\mathbb{k}[X]$ to the field of fractions $\mathbb{k}(X)$. This is a subfield of $\mathbb{k}(X)$ which consists of all rational $\operatorname{SAut}(\mathrm{X})$-invariants. If it is trivial i.e., if $\operatorname{FML}(X)=\mathbb{k}$, then so is $\operatorname{ML}(X)$, while the converse is not true in general. Triviality of $\operatorname{FML}(X)$ is equivalent to the existence of a flexible point in $X_{\text {reg }}$, and to the existence of an open $\operatorname{SAut}(X)$-orbit in $X$.

The question arises how these invariants are connected with rationality properties of the variety $X$. There are examples of non-unirational affine threefolds $\mathrm{X}$ with $\operatorname{ML}(X)=$ $\mathbb{k}$ birationally equivalent to $C \times \mathbb{A}^{2}$, where $C$ is a curve of genus $g \geq 1$, see [20, Example 4.2]. For such a threefold $X$ the general SAut $(X)$-orbits have dimension two, the field Makar-Limanov invariant $\operatorname{FML}(X)$ is non-trivial, and there is no flexible point in $X$.

The next proposition confirms, in particular, Conjecture 5.3 in [21] (cf. also [4] and [24]).

Proposition 3.1. [1, Proposition 5.1] Let $X$ be an irreducible affine variety $X$. If the field Makar-Limanov invariant $F M L(X)$ is trivial then $X$ is unirational. 
Indeed, the condition $\operatorname{FML}(X)=\mathbb{k}$ implies that the group $\operatorname{SAut}(X)$ acts on $X$ with an open orbit $O$. Thus there are $\mathbb{G}_{a}$-subgroups $H_{1}, \ldots, H_{s}$ in $\operatorname{SAut}(X)$ and a point $x \in X$ such that the image of the map

$$
H_{1} \times \ldots \times H_{s} \rightarrow X, \quad\left(h_{1}, \ldots, h_{s}\right) \mapsto\left(h_{1} \ldots h_{s}\right) \cdot x
$$

coincides with $O$. Since $H_{1} \times \ldots \times H_{s}$ is isomorphic (as a variety) to the affine space $\mathbb{A}^{s}$, this yields unirationality of $X$. Moreover, any two points in $O$ are contained in the image of a morphism $\mathbb{A}^{1} \rightarrow O$. In particular, $O$ is $\mathbb{A}^{1}$-connected in the sense of $[13$, $6.2]$.

In general, flexibility implies neither rationality nor stable rationality. Indeed, there exists a finite subgroup $F \subset \mathrm{SL}_{n}$, where $n \geq 4$, such that the smooth unirational affine variety $X=\mathrm{SL}_{n} / F$ is not stably rational, see [24, Example 1.22]. However, by 2.3 the variety $X$ is flexible and the group $\operatorname{SAut}(X)$ acts infinitely transitively on $X$.

We expect further development of the invariant theory for algebraically generated groups.

\section{Geometric consequences}

Let us start with several results related to Theorem 1.1.

4.1. Collective transitivity. By a collective infinite transitivity we mean a possibility to move simultaneously (that is, by the same automorphism) an arbitrary finite set of points along their orbits into a given position. We illustrate our general results in this direction on a concrete example from linear algebra, cf. [27].

Let $X=\operatorname{Mat}(n, m)$ be the space of all $n \times m$ matrices over $\mathbb{k}$. The subset $X_{r} \subseteq X$ of matrices of rank $r$ is well known to have dimension $m n-(m-r)(n-r)$. In the following we always assume that this dimension is $\geq 2$. The product $\mathrm{SL}_{n} \times \mathrm{SL}_{m}$ acts on $X$ via the left-right multiplication preserving the strata $X_{r}$. For every $k \neq l$ we let $E_{k l} \in \mathfrak{s l}_{n}$ and $E^{k l} \in \mathfrak{s l}_{m}$ denote the nilpotent matrices with $x_{k l}=1$ and the other entries equal zero. Let further $H_{k l}=I_{n}+\mathbb{k} E_{k l} \subseteq \mathrm{SL}_{n}$ and $H^{k l}=I_{m}+\mathbb{k} E^{k l} \subseteq \mathrm{SL}_{m}$ be the corresponding one-parameter unipotent subgroups acting on the stratification $X=\bigcup_{r} X_{r}$, and let $\delta_{k l}$ and $\delta^{k l}$, respectively, be the corresponding locally nilpotent vector fields on $X$ tangent to the strata.

We call elementary the one-parameter unipotent subgroups $H_{k l}, H^{k l}$, and all their replicas. In the following theorem we establish the collective infinite transitivity on the above stratification of the subgroup $G$ of $\operatorname{SAut}(X)$ generated by the two sides elementary subgroups.

By a well known theorem of linear algebra, the subgroup $\mathrm{SL}_{n} \times \mathrm{SL}_{m} \subseteq G$ acts transitively on each stratum $X_{r}$ (and so these strata are $G$-orbits) except for the open stratum $X_{n}$ in the case where $m=n$. In the latter case the $G$-orbits contained in $X_{n}$ are the level sets of the determinant.

Theorem 4.1. [1, Theorem 3.3] Given two finite ordered collections $\mathcal{B}$ and $\mathcal{B}^{\prime}$ of distinct matrices in $\operatorname{Mat}(n, m)$ of the same cardinality, with the same sequence of ranks, and in the case where $m=n$ with the same sequence of determinants, we can simultaneously transform $\mathcal{B}$ into $\mathcal{B}^{\prime}$ by means of an element $g \in G$, where $G \subseteq \operatorname{SAut}(\operatorname{Mat}(n, m))$ is the subgroup generated by all elementary one-parameter unipotent subgroups. 
See [1, Section 3.3] for similar results on symmetric and skew-symmetric matrices.

4.2. $\mathbb{A}^{1}$-richness. Let $X$ be a flexible affine variety of dimension $\geq 2$, and let $p_{1}, \ldots, p_{k} \in X_{\text {reg }}$ be a $k$-tuple. Fix a $\mathbb{G}_{a}$-orbit $C$ on $X$ and some $k$-tuple of distinct points $q_{1}, \ldots, q_{k} \in O$. Due to infinite transitivity there is an element $g \in \operatorname{SAut}(X)$ such that $g \cdot q_{1}=p_{1}, \ldots, g \cdot q_{k}=p_{k}$. So the translate $g \cdot C$ of $C$ is a $\mathbb{G}_{a}$-orbit on $X$ passing through $p_{1}, \ldots, p_{k}$. This elementary observation can be strengthened in the following way.

An affine variety $X$ is called $\mathbb{A}^{1}$-rich if for every finite subset $Z$ and every algebraic subset $Y$ of codimension $\geq 2$ there is a curve in $X$ isomorphic to the affine line $\mathbb{A}^{1}$, which is disjoint with $Y$ and passes through every point of $Z$ ([15]).

The following result is immediate from the Transversality Theorem 3.1.

Theorem 4.1. [1, Corollary 4.18] Let $X$ be an affine variety. Suppose that the group $\operatorname{SAut}(X)$ acts with an open orbit $O \subseteq X$. Then for any finite subset $Z \subseteq O$ and for any closed subset $Y \subseteq X$ of codimension $\geq 2$ with $Z \cap Y=\varnothing$ there is an orbit $C \cong \mathbb{A}^{1}$ of $a \mathbb{G}_{a}$-action on $X$ which does not meet $Y$ and passes through each point of $Z$.

In the special case where $X=\mathbb{A}_{\mathbb{C}}^{n}$ this also follows from the Gromov-Winkelmann Theorem [30] which says that the group $\operatorname{Aut}\left(\mathbb{A}^{n} \backslash Y\right)$ acts transitively on $\mathbb{A}^{n} \backslash Y$, combined with the equivalence of transitivity and infinite transitivity of Theorem 1.1, which is valid in this setting as well. More generally, we also show that $C$ as in the theorem can be chosen to have prescribed jets at the points of $Z$.

4.3. Prescribed jets of automorphisms. Our results on infinite transitivity may be strengthened in the following way, see [1, Theorem 4.14 and Remark 4.16].

Theorem 4.2. Let $X$ be a flexible affine variety of dimension $n \geq 2$ equipped with an algebraic volume form ${ }^{1} \omega$. Then for any $m \geq 0$ and for any finite subset $Z \subseteq X_{\text {reg }}$ there exists an automorphism $g \in \operatorname{SAut}(X)$ with prescribed $m$-jets at the points $p \in Z$, provided these jets preserve $\omega$ and inject $Z$ into $X_{\text {reg. }}$. The same holds without the requirement that there is a global volume form on $X_{\text {reg }}$ provided that for every $p \in Z$ the corresponding jet fixes the point $p$ and its linear part belongs to the group $\operatorname{SL}\left(T_{p} X\right)$.

4.4. The Oka-Grauert-Gromov Principle for flexible varieties. Let us provide an important application of flexibility in analytic geometry; see [1, Theorem 6.2 and Proposition 6.3]. We address the reader to [1, §6] for more details and a survey.

Theorem 4.3. Let $\pi: X \rightarrow B$ be a surjective submersion of smooth irreducible affine algebraic varieties over $\mathbb{C}$ such that for some algebraically generated subgroup $G \subseteq$ $\operatorname{Aut}(X)$ the orbits of $G$ coincide with the fibers of $\pi$. Then the Oka-Grauert-Gromov principle holds for $\pi: X \rightarrow B$. That is, any continuous section of $\pi$ is homotopic to a holomorphic one, and any two holomorphic sections of $\pi$ that are homotopic via continuous sections are also homotopic via holomorphic ones.

\section{Open PROBLEMS}

Let us finish this note with several open problems on flexible varieties. The examples from subsection 2.4 motivate the following problem.

\footnotetext{
${ }^{1}$ By this we mean a nowhere vanishing $n$-form defined on $X_{\text {reg }}$.
} 
Characterize flexible varieties among the normal almost homogeneous affine varieties.

By the result described in 2.4.1, a smooth almost homogeneous variety is flexible. In fact, in all examples that we know an almost homogeneous normal variety is flexible. For instance, one might hope for positive results in the class of spherical varieties. By definition, a $G$-variety $X$ is spherical if a Borel subgroup $B$ of $G$ acts on $X$ with an open orbit. An important particular case is the variety $X=\operatorname{Spec} \mathbb{k}[G / U]$, where $U$ is a maximal unipotent subgroup of a semisimple group $G$.

To formulate the next problem we need to introduce some more notation. Let $Y$ be a closed subvariety of an affine variety $X$. Denote by $\operatorname{SAut}(X)_{Y}$ the subgroup generated by all one-parameter unipotent subgroups $\exp (\mathbb{k} \partial)$, where the locally nilpotent vector field $\partial$ vanishes on $Y$.

Assume that the group $\operatorname{SAut}(X)$ acts on $X$ with an open orbit $O$, and let $Y \subseteq O$ be a closed subvariety of codimension $\geq 2$. Is it true that the group $\operatorname{SAut}(X)_{Y}$ acts on $O \backslash Y$ transitively? In particular, is $X \backslash Y$ flexible if so is $X$ ?

Some positive results on this problem can be found in [1, Proposition 4.19].

Our last problem concerns exotic structures on the affine spaces.

Does there exist a flexible exotic algebraic structure on an affine space that is, a flexible smooth affine algebraic variety over $\mathbb{C}$ diffeomorphic but not isomorphic to an affine space $\mathbb{A}_{\mathbb{C}}^{n}$ ?

Notice that for all the exotic structures on $\mathbb{A}_{\mathbb{C}}^{n}$ constructed so far, the Makar-Limanov invariant is non-trivial, whereas for a flexible such structure even the field MakarLimanov invariant must be trivial (cf. however [8]).

\section{REFERENCES}

[1] I.V. Arzhantsev, H. Flenner, S. Kaliman, F. Kutzschebauch, M. Zaidenberg. Flexible varieties and automorphism groups. Duke Math. J., to appear; arXiv:1011.5375, 41 p.

[2] I.V. Arzhantsev, K. Kuyumzhiyan, M. Zaidenberg. Flag varieties, toric varieties, and suspensions: three instances of infinite transitivity. Sbornik: Math. 203 (2012), no. 7, 3-30.

[3] V. Batyrev, F. Haddad. On the geometry of SL(2)-equivariant flips. Mosc. Math. J. 8 (2008), 621-646.

[4] F. Bogomolov, I. Karzhemanov, K. Kuyumzhiyan. Unirationality and existence of infinitely transitive models. arXiv:1204.0862, 9p.

[5] A. Borel. Les bouts des espaces homogènes de groupes de Lie. Ann. Math. (2) 58 (1953), 443-457.

[6] V. I. Danilov, M. H. Gizatullin. Examples of nonhomogeneous quasihomogeneous surfaces. Math. USSR Izv. 8 (1974), 43-60.

[7] A. Dubouloz. Completions of normal affine surfaces with a trivial Makar-Limanov invariant. Michigan Math. J. 52 (2004), 289-308.

[8] A. Dubouloz. The cylinder over the Koras-Russell cubic threefold has a trivial Makar-Limanov invariant. Transform. Groups 14 (2009), 531-539.

[9] H. Flenner, S. Kaliman, M. Zaidenberg. Smooth Affine Surfaces with Non-Unique $\mathbb{C}^{*}$-Actions. J. Algebraic Geom. 20 (2011), 329-398.

[10] G. Freudenburg. Algebraic Theory of Locally Nilpotent Derivations. Encyclopaedia of Mathematical Sciences, Vol. 136, Springer-Verlag, 2006.

[11] M. H. Gizatullin. Affine surfaces that can be augmented by a nonsingular rational curve (Russian). Izv. Akad. Nauk SSSR Ser. Mat. 34 (1970), 778-802.

[12] M. H. Gizatullin. Quasihomogeneous affine surfaces. USSR Izv. 5 (1971), 1057-1081. 
[13] S. Kaliman, F. Kutzschebauch. Criteria for the density property of complex manifolds. Invent. Math. 172 (2008), 71-87.

[14] S. Kaliman, M. Zaidenberg. Affine modifications and affine hypersurfaces with a very transitive automorphism group. Transform. Groups 4 (1999), 53-95.

[15] S. Kaliman, M. Zaidenberg. Miyanishi's characterization of the affine 3-space does not hold in higher dimensions. Ann. Inst. Fourier (Grenoble) 50 (2000), 1649-1669.

[16] T. Kishimoto, Yu. Prokhorov, M. Zaidenberg. Group actions on affine cones. CRM Proceedings and Lecture Notes, Vol. 54, Amer. Math. Soc., 2011, pp. 123-164.

[17] T. Kishimoto, Yu. Prokhorov, M. Zaidenberg. Unipotent group actions on del Pezzo cones. (Under preparation).

[18] F. Knop. Mehrfach transitive Operationen algebraischer Gruppen. Arch. Math. 41 (1983), 438446.

[19] K. Kuyumzhiyan, F. Mangolte. Infinitely transitive actions on real affine suspensions. J. Pure Appl. Algebra 216 (2012) no. 10, 2106-2112.

[20] A. Liendo. Affine $T$-varieties of complexity one and locally nilpotent derivations. Transform. Groups 15 (2010), 389-425.

[21] A. Liendo. $\mathbb{G}_{a}$-actions of fiber type on affine $T$-varieties. J. Algebra 324 (2010), 3653-3665.

[22] A.Yu. Perepechko. Flexibility of affine cones over del Pezzo surfaces of degree 4 and 5. Funct. Anal. Appl., to appear; arXiv: 1108.5841, 6 p.

[23] V.L. Popov. Quasihomogeneous affine algebraic varieties of the group SL(2). Math. USSR Izv. 7 (1973), 793-831.

[24] V.L. Popov. On the Makar-Limanov, Derksen invariants, and finite automorphism groups of algebraic varieties. CRM Proceedings and Lecture Notes, Vol. 54, Amer. Math. Soc., 2011, pp. 289-311.

[25] V.L. Popov, E.B. Vinberg. On a certain class of quasihomogeneous affine varieties. Math. USSR Izv. 6 (1972), 743-758.

[26] C. P. Ramanujam. A note on automorphism groups of algebraic varieties. Math. Ann. 156 (1964), 25-33.

[27] Z. Reichstein. I. On automorphisms of matrix invariants. Trans. Amer. Math. Soc. 340 (1993), 353-371; II. On automorphisms of matrix invariants induced from the trace ring. Linear Algebra Appl. 193 (1993), 51-74.

[28] I. R. Shafarevich. On some infinite-dimensional groups. Rend. Mat. Appl. (5) 25 (1966), no. 1-2, 208-212.

[29] I. P. Shestakov, U. U. Umirbaev. The tame and the wild automorphisms of polynomial rings in three variables. J. Amer. Math. Soc. 17 (2004), 197-227.

[30] J. Winkelmann. On automorphisms of complements of analytic subsets in $\mathbb{C}^{n}$. Math. Z. 204 (1990), 117-127.

Department of Algebra, Faculty of Mechanics and Mathematics, Moscow State University, Leninskie Gory 1, GSP-1, Moscow, 119991, Russia

E-mail address: arjantse@mccme.ru

Fakultät für Mathematik, Ruhr Universität Bochum, Geb. NA 2/72, UniversitätsSTR. 150, 44780 BoChum, Germany

E-mail address: Hubert.Flenner@rub.de

Department of Mathematics, University of Miami, Coral Gables, FL 33124, USA

E-mail address: kaliman@math.miami.edu

Mathematisches Institut, Universität Bern, Sidlerstrasse 5, CH-3012 Bern, SwitzerLAND,

E-mail address: frank.kutzschebauch@math.unibe.ch 
Université Grenoble I, Institut Fourier, UMR 5582 CNRS-UJF, BP 74, 38402 St. Martin d'Hères CÉdex, France

E-mail address: Mikhail.Zaidenberg@ujf-grenoble.fr 